\title{
Experimental Investigation of Wire Electrical Discharge Machining of NdFeB Permanent Magnets with an RC-Type Machine
}

\author{
Jeremy Greer, Andrew J. Petruska, Arthur W. Mahoney, Manikantan Nambi, Eberhard Bamberg, and Jake J. Abbott
}

(Submitted April 25, 2013; in revised form December 12, 2013; published online January 1, 2014)

\begin{abstract}
This paper focuses on wire electrical discharge machining (WEDM) of devices made from commercially available sintered neodymium-iron-boron $(\mathrm{NdFeB})$ rare-earth magnets that have been magnetized before machining. We conduct an experimental study to quantify the effects of the voltage and capacitance of an RC-type WEDM machine, as well as the magnet polarity, on slicing rate, mean kerf, and variation in kerf. We find that voltage and capacitance affect slicing rate, that voltage affects mean kerf, that no parameters tested affect the variation in kerf, and that the polarity of the magnet being machined does not affect the machining process. Linear regression is used to fit simple predictive models to the data with good agreement. Further analysis is performed to characterize the loss in permanent magnetization on the surface of the magnet due to heating during the machining process, and it is found that the outer layer of approximately $35 \mu \mathrm{m}$ in depth is demagnetized when using the highest voltage and capacitance values tested.
\end{abstract}

Keywords demagnetization, EDM, kerf, magnetization, slicing rate

\section{Introduction}

There has been significant research in the area of microscale and mesoscale "robotic" devices for future minimally invasive medical applications, such as targeted delivery of therapies and removal of vessel blockages (Ref 1). A large variety of these proposed devices have utilized magnetic fields for propulsion and control of helical structures, including screws for use in soft tissue and lumens, and bacteria-inspired swimmers for use in fluid environments. Prior work has typically considered a magnetic element attached to a nonmagnetic helical structure, with the result that the magnetic torque available for propulsion is smaller than what would be achievable if more of the device's volume could carry a magnetization. This motivates our problem: can we create magnetic devices such as screws and helical swimmers from high-grade diametrically magnetized permanent magnets, such that a significant portion of the structure holds a magnetization?

In this paper, we investigate the process of machining a sintered rare-earth magnet that is already magnetized, using

Jeremy Greer, Andrew J. Petruska, Manikantan Nambi, and Jake J. Abbott, Department of Mechanical Engineering, University of Utah, 50 S. Central Campus Dr., Salt Lake City, UT 84112, USA; Arthur W. Mahoney, School of Computing, University of Utah, $50 \mathrm{~S}$. Central Campus Dr., Salt Lake City, UT 84112, USA; and Eberhard Bamberg, Viteris Technologies, 1883 S. 5070 W., Salt Lake City, UT 84112, USA. Contact e-mails: jeremy.greer.eng@gmail.com, andrew. petruska@utah.edu, art.mahoney@utah.edu,m.nambi@utah.edu, eberhard.bamberg@viteris.com, and jake.abbott@utah.edu. wire electrical discharge machining (WEDM) with an RC-type machine. The magnet we consider is a neodymium-iron-boron (NdFeB) type magnet with a nickel-copper-nickel coating to inhibit corrosion; this type of high-grade permanent magnet is readily available commercially in a variety of geometries down to size of approximately $1 \mathrm{~mm}$ in characteristic length. WEDM is an advantageous choice over conventional machining methods because it is a thermal process and not a mechanical process; this is useful for machining sintered $\mathrm{NdFeB}$ magnets, which exhibit high hardness (Ref 2), low ductility, and a tensile strength of approximately $100 \mathrm{MPa}$ (Ref 3). Material removal mechanisms for EDM of $\mathrm{NdFeB}$ have been identified previously as melting and evaporation, thermal cracking, and wholegrain spalling (Ref 4). WEDM can also yield a high degree of dimensional accuracy, using wire sizes down to $20 \mu \mathrm{m}$, with the width of cut, known as kerf, slightly larger depending on machine parameters. It has been shown that EDM machining of $\mathrm{NdFeB}$ can also improve corrosion resistance by forming an amorphous surface layer (Ref 5).

Initial test cutting and published research (Ref 3) have shown that WEDM of NdFeB is relatively slow. Work has been done to increase the cutting rate by controlling the spark cycle on a MOSFET-type machine (Ref 6), and by ultrasonic vibration of the tool, which also reduces the recast layer (Ref 7) and leads to a finer surface finish (Ref 8); however, these techniques are not available on the RC-type WEDM machine of interest here. Therefore, the first objective of this research was to study the effect of the control parameters for an RC-type WEDM machine (voltage and capacitance), as well as the direction of magnetic polarity of the workpiece, on the cutting of $\mathrm{NdFeB}$ in terms of slicing rate, kerf, and variation in kerf. A Taguchi analysis is used here, similar to other experiments done for any type of machining process (Ref 9). After determining which parameters have an effect on the three cutting parameters of interest, linear regression is performed to provide a model that relates the input parameters to the output parameters. 
When the magnet is heated above its Curie temperature, it loses its magnetization (Ref 10). Since a portion of the magnet being machined in WEDM is necessarily heated above the Curie temperature to be melted off, some portion of the magnet must also suffer a loss of the permanent magnetization. Most magnets are manufactured net shape, and when WEDM is used to further shape the magnet, it is usually magnetized postmachining (Ref 11). Thermal stresses in NdFeB induced by WEDM have been modeled previously (Ref 12). There is recent evidence that surface magnetization decreases as the power being discharged with a MOSFET-type machine is increased (Ref 13), and the same group measured the temperature distribution in the magnet as it is being machined (Ref 14); however, it is difficult to accurately determine the amount of demagnetized material after machining, based on the data reported. Therefore, the second objective of this research was to determine the magnetic loss due to heat from machining of the magnet using the RC-type WEDM machine. A finite element analysis (FEA) and experimental measurements are combined here to estimate the extent of the demagnetized layer in the permanent magnet. If the demagnetization of a given machined part can be determined to be sufficiently small, then the part need not be remagnetized after machining (which may require significant capital equipment and custom fixturing).

\section{Characterization of cutting parameters}

\subsection{Methods}

The WEDM machine used for this research is an RC-type machine constructed at the University of Utah (Ref 15). It was primarily designed to be used for manufacturing components for miniature mechanical systems and microelectromechanical systems (MEMS), and has also been used in research of cutting of semiconductor materials such as germanium (Ref 16) and silicon (Ref 17). The machine can accommodate small $(20 \mu \mathrm{m})$ diameter wires. The spark parameters can be adjusted between 0 and $300 \mathrm{~V}$, and a wide range of capacitors can also be used, commonly 3.3-33 nF. A discharge resistor of $36 \Omega$ limits the current to $120 \mathrm{~mA}$. A commercial version of this system is available through Viteris Technologies.

A notable feature of the machine's control system is the ability to adaptively optimize the feed rate of the machine by examining the ratio between the frequency of shorts and the frequency of sparks of the wire. In order for sparking and subsequent machining to take place, the spark gap between the wire and the workpiece must be maintained. If the wire contacts the workpiece, the circuit is shorted and no material is removed. Most commercial machines set feed rates sufficiently slow that the wire never shorts on the workpiece; this approach works, but may be slow. The machining rates are usually determined from experience and knowledge of the material being machined and the manufacture's tabulated values. The control software of this machine adjusts the speed by detecting when shorts occur. When the machine shorts, it must back up to re-approach the spark gap. This reduces the overall feed rate and increases machining time. By slowing the feed to an optimal ratio of shorts and sparks, it allows the machine to feed at the fastest possible rate for the given input parameters. The optimizedfeed-rate control system allows exotic materials to be used when there is not much initially known about the cutting characteristics of the material, and more importantly for this paper, it allows us to characterize the cutting characteristics of an exotic material, namely sintered $\mathrm{NdFeB}$.

A design of experiments (DOE) was planned using the Taguchi methodology to determine which control variables have a significant effect (along with their relative impact) on the cutting parameters of interest (Ref 9). Taguchi DOE can greatly reduce the number of experiments required compared to a full factorial design, and is particularly useful when experiments are costly. In the case of this experiment, it reduced the number of experiments from 27 for a full factorial design to 9 for the Taguchi DOE. Similar DOE's have been performed for the analysis of WEDM parameters for gallium doped p-type germanium (Ref 18).

The orthogonal array used for this experiment is the L9 array, which can be used for experiments on as many as four factors of three levels, although four factors are not necessary for proper Taguchi analysis. The control parameters for this experiment are the machine's capacitance and voltage, and polar direction of the permanent-magnet workpiece being machined (relative to the wire). The output parameters used for this experiment are the slicing rate, kerf (quantified with the mean of kerf measurements of a given cut), and variation in kerf (quantified with the standard deviation of kerf measurements of a given cut).

The slicing rate is the product of feed rate and workpiece thickness. It is measured in area/time, in this case $\mathrm{mm}^{2} / \mathrm{min}$. In order to determine the volumetric material removal rate, one would multiply the slicing rate by the kerf.

The polar direction of the permanent magnet is the direction from the south pole to the north pole of the magnet in the coordinate system of the WEDM machine. The polar direction is of particular interest because of the force on the current carrying wire, called the Lorentz force, governed by $\vec{F}=L(\vec{i} \times \vec{B})$, where $\vec{F}$ \{newton $\}$ is the force vector on the wire, $L$ \{meter\} is the scalar length of wire in the field, $\vec{i}$ \{ampere\} is the current vector, and $\vec{B}$ \{tesla\} is the magnetic field vector at the location of the wire. This effect would cause the EDM wire to deflect and possibly vibrate while cutting due to the current from the discharges in the wire interacting with the magnetic field of the sample. Indications of this effect could be slow machining rates due to a high number of shorts in the wire, or larger than normal kerf due to the wire deflecting and vibrating.

The Taguchi DOE includes three levels for each of the three input variables (i.e., control factors). The WEDM voltages considered here are 150,250, and $300 \mathrm{~V}$. Although the voltage on the WEDM machine used can be set at any value between 0 $300 \mathrm{~V}$, the range of $150-300 \mathrm{~V}$ was considered most relevant.

Table 1 Taguchi design of experiment

\begin{tabular}{lccc}
\hline & Voltage $(\mathbf{V})$ & Capacitance $(\mathbf{n F})$ & Polar direction \\
\hline 1 & 150 & 3.3 & $X$ \\
2 & 150 & 10 & $Y$ \\
3 & 150 & 22 & $Z$ \\
4 & 250 & 3.3 & $Y$ \\
5 & 250 & 10 & $Z$ \\
6 & 250 & 22 & $X$ \\
7 & 300 & 3.3 & $Z$ \\
8 & 300 & 10 & $X$ \\
9 & 300 & 22 & $Y$
\end{tabular}


The capacitance values considered here are $3.3,10$, and $22 \mathrm{nF}$. Commercially available capacitor values limit the available choices, and preliminary testing showed that higher capacitance values than $22 \mathrm{nF}$ contributed to excessive wire breakage. For the third control factor, the magnet polarity was considered in three mutually orthogonal directions $(X, Y, Z)$, where the EDM wire is aligned with the $X$ direction (see Greer, Ref 19, for more detail). The final Taguchi DOE using the L9 array is shown in Table 1.

Experiments were performed on $9.53 \mathrm{~mm}$ (0.375 in.) cubic grade-N42 NdFeB magnets. Cuts were completed by cutting deep slits halfway through the cube, near the center of the cube, as shown in Fig. 1. The wire position was sampled and recorded every second and written to a text file by the WEDM software. This information was then used to calculate the slicing rate of each cut. Nonmagnetic $100-\mu \mathrm{m}$-diameter brass wire was used for all cuts in the experiment. The kerf values were measured on an optical measurement system. Each slice was measured 20 times and the mean and standard deviation of kerf were recorded for each slice. Each experiment was performed three times as shown in Fig. 1.

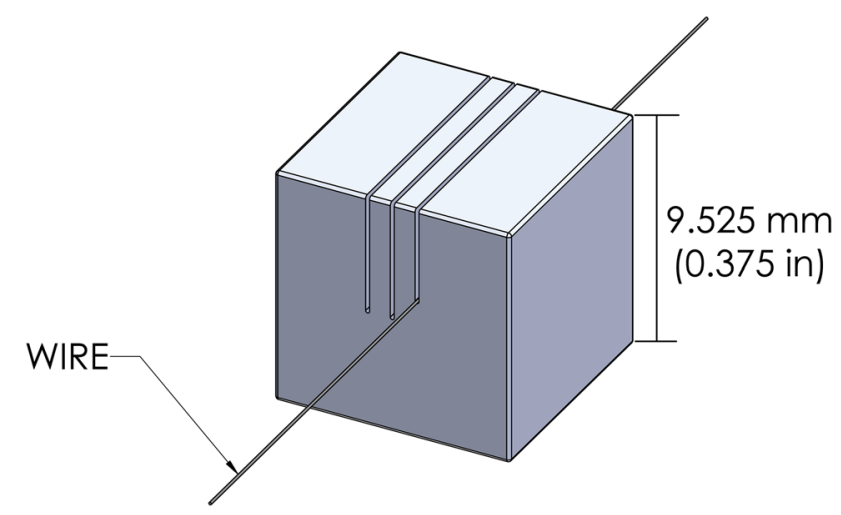

Fig. 1 Illustration of cuts for experiments

Table 2 Experimental data for slicing rate

\begin{tabular}{lccccc}
\hline $\begin{array}{l}\text { Voltage } \\
\text { (V) }\end{array}$ & $\begin{array}{c}\text { Capacitor } \\
\text { (nF) }\end{array}$ & $\begin{array}{c}\text { Magnet } \\
\text { polarity }\end{array}$ & $\begin{array}{c}\text { Slicing } \\
\text { rate 1 } \\
\left(\mathbf{m m}^{2} / \mathbf{m i n}\right)\end{array}$ & $\begin{array}{c}\text { Slicing } \\
\text { rate 2 } \\
\left(\mathbf{m m}^{\mathbf{2}} / \mathbf{m i n}\right)\end{array}$ & $\begin{array}{c}\text { Slicing } \\
\text { rate 3 } \\
\left(\mathbf{m m}^{2} / \mathbf{m i n}\right)\end{array}$ \\
\hline 150 & 3.3 & $X$ & 0.222 & 0.271 & 0.213 \\
150 & 10 & $Y$ & 0.442 & 0.438 & 0.433 \\
150 & 22 & $Z$ & 0.674 & 0.628 & 0.682 \\
250 & 3.3 & $Y$ & 0.418 & 0.446 & 0.445 \\
250 & 10 & $Z$ & 0.687 & 0.636 & 0.616 \\
250 & 22 & $X$ & 0.929 & 1.067 & 0.953 \\
300 & 3.3 & $Z$ & 0.433 & 0.469 & 0.409 \\
300 & 10 & $X$ & 0.677 & 0.778 & 0.641 \\
300 & 22 & $Y$ & 1.223 & 1.353 & 1.256 \\
\hline
\end{tabular}

Analysis of variance (ANOVA) of a Taguchi L9 experiment is performed to determine the influence of each input parameter on the output variables of interest (Ref 9). First, the sum of squares $\left(S S_{i}\right)$ for each input parameter $(i)$ is calculated by summing the squared difference between the mean output at a particular level of the parameter and the total mean for the experiment. Similarly, the sum of squares for the total experiment $\left(S S_{T}\right)$ is calculated. The sum of squares of the error $\left(S S_{E}\right)$ is given by the difference between $S S_{T}$ and $S S_{i}$. The mean square $\left(M S_{i}\right)$, which is an estimate of the variance, is then calculated by the dividing $S S_{i}$ by its degrees of freedom $(n-1)$, where $n$ is the number of levels of an input parameter. The $F$ ratio is calculated by dividing the $M S_{i}$ of each input parameter by the mean square of error (MSE). The contribution of each parameter $i$ is calculated by the ratio of $S S_{i} / S S_{T}$. The result of the ANOVA is the percentage contribution of the total variance in the data that is accounted for by each of the three input parameters, along with the probability that each parameter has a statistically significant effect on the output variable of interest.

Based on the results of the ANOVA, we perform a regression analysis to find simple equations that describe the output parameters as simple functions of the input parameters. We consider models that include each of the statistically significant input parameters as main effects, as well as their interactions. We perform a regression fit, remove the single least-significant term, redo the regression fit on the reduced model, and repeat until all remaining terms in the model have a significance of $p<0.05$. The ANOVA and regression analysis was performed using SPSS Statistics 20, Release Version 20.0.0 (IBM Corp., 2011, Armonk, NY).

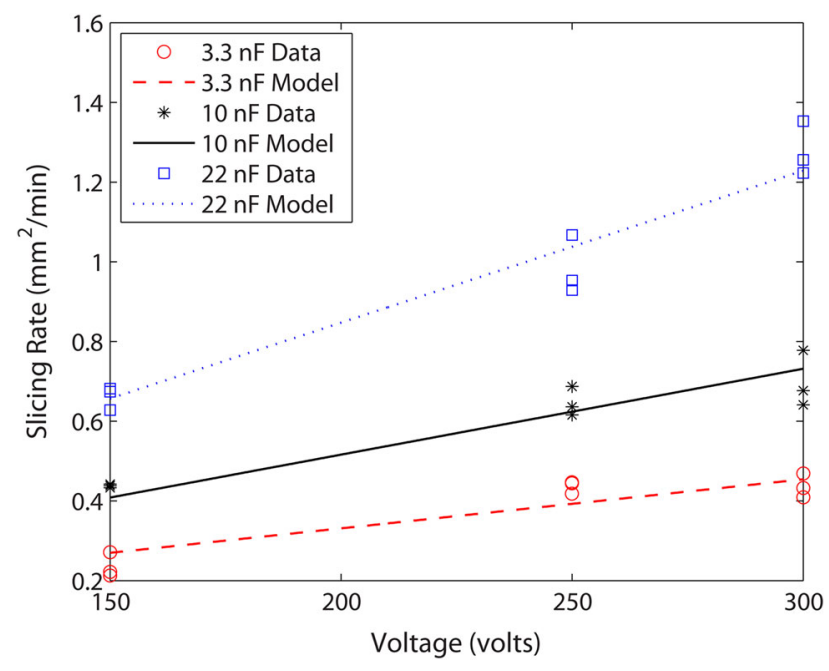

Fig. 2 Regression fit for slicing rate as a function of capacitance and voltage $\left(R^{2}=0.994\right)$

Table 3 ANOVA for slicing rate

\begin{tabular}{|c|c|c|c|c|c|c|}
\hline Factor & DoF & Sum of squares & Mean square & $F$ ratio & $p$ value & Contribution (\%) \\
\hline Voltage & 2 & 0.2021 & 0.1011 & 8.045 & 0.1038 & 24.8 \\
\hline Capacitance & 2 & 0.5598 & 0.2799 & 22.29 & 0.0421 & 68.7 \\
\hline Polar direction & 2 & 0.02778 & 0.01389 & 1.106 & 0.4884 & 3.4 \\
\hline Residual & 2 & 0.02512 & 0.01256 & & & 3.1 \\
\hline Total & 8 & 0.8149 & & & & \\
\hline
\end{tabular}


Table 4 Experimental data for mean kerf

\begin{tabular}{lccccc}
\hline Voltage $(\mathbf{V})$ & Capacitor $(\mathbf{n F})$ & Magnet polarity & Mean Kerf $\mathbf{1}(\boldsymbol{\mu m})$ & Mean Kerf 2 $(\boldsymbol{\mu m})$ & Mean Kerf 3 $(\boldsymbol{\mu m})$ \\
\hline 150 & 3.3 & $X$ & 138.7 & 134.6 & 134 \\
150 & 10 & $Y$ & 137.1 & 133.6 & 132.9 \\
150 & 22 & $Z$ & 131.6 & 156.8 & 13.2 \\
250 & 3.3 & $Y$ & 152.5 & 158.6 & 153.8 \\
250 & 10 & $Z$ & 156.8 & 163.2 & 156.9 \\
250 & 22 & $X$ & 163.7 & 161.1 & 162.3 \\
300 & 3.3 & $Z$ & 162.2 & 169.2 & 163.8 \\
300 & 10 & $X$ & 169.9 & 170.7 \\
\hline 00 & 22 & $Y$ & & & \\
\hline
\end{tabular}

Table 5 ANOVA for mean kerf

\begin{tabular}{|c|c|c|c|c|c|c|}
\hline Factor & df & Sum of squares & Mean square & $F$ ratio & $p$ value & Contribution (\%) \\
\hline Voltage & 2 & 1449 & 724.4 & 54 & 0.0182 & 97.1 \\
\hline Capacitance & 2 & 2.614 & 1.307 & 0.0974 & 0.9112 & 0.2 \\
\hline Polar direction & 2 & 14.1 & 7.051 & 0.5257 & 0.6555 & 0.9 \\
\hline Residual & 2 & 26.83 & 13.41 & & & 1.8 \\
\hline Total & 8 & 1492 & & & & \\
\hline
\end{tabular}

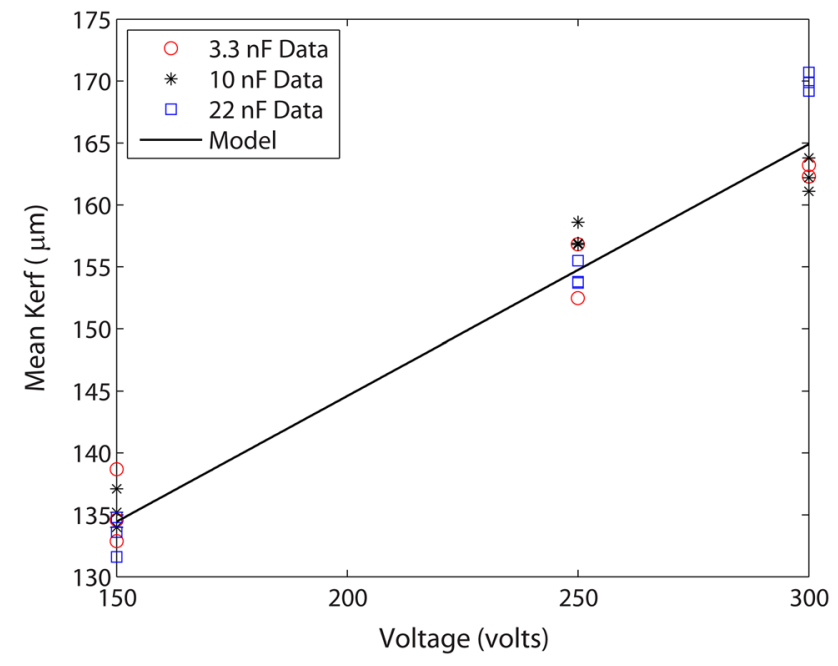

Fig. 3 Regression fit for mean kerf as a function of voltage $\left(R^{2}=0.959\right)$

\subsection{Results}

The results for the slicing-rate experiment are found in Table 2, and the ANOVA results are found in Table 3. For slicing rate, the capacitance is the primary contributing factor $(68.7 \%, p=0.0421)$, voltage is secondary but with marginal statistical significance $(24.8 \%, p=0.1038)$, and the polar direction plays a statistically insignificant role $(3.4 \%$, $p=0.4884$ ) that is roughly equivalent to the residual noise in the data $(3.1 \%)$. We performed a regression analysis on slicing rate, based on the two input parameters that substantially influence slicing rate: voltage and capacitance. The result, shown in Fig. 2, is an equation for slicing rate $R_{\mathrm{S}}\left\{\mathrm{mm}^{2} / \mathrm{min}\right\}$ that is a simple function of voltage $V$ \{volts $\}$ and capacitance $C$ $\{\mathrm{nF}\}$ that is a good fit in the region of the data collected:
Table 6 Experimental data for standard deviation in kerf

\begin{tabular}{lccccc}
\hline $\begin{array}{l}\text { Voltage } \\
(\mathbf{V})\end{array}$ & $\begin{array}{c}\text { Capacitor } \\
(\mathbf{n F})\end{array}$ & $\begin{array}{c}\text { Magnet } \\
\text { polarity }\end{array}$ & $\begin{array}{c}\text { Kerf } \boldsymbol{\sigma} \mathbf{1} \\
(\boldsymbol{\mu} \mathbf{m})\end{array}$ & $\begin{array}{c}\text { Kerf } \boldsymbol{\sigma} 2 \\
(\boldsymbol{\mu} \mathbf{m})\end{array}$ & $\begin{array}{c}\text { Kerf } \boldsymbol{~ 3 ~} \\
(\boldsymbol{\mu} \mathbf{m})\end{array}$ \\
\hline 150 & 3.3 & $X$ & 2.5 & 1.2 & 2.9 \\
150 & 10 & $Y$ & 2.7 & 2.5 & 1.9 \\
150 & 22 & $Z$ & 2.3 & 2.2 & 2.2 \\
250 & 3.3 & $Y$ & 2.4 & 1.4 & 1.6 \\
250 & 10 & $Z$ & 1.6 & 2.6 & 1.4 \\
250 & 22 & $X$ & 2.2 & 3.1 & 1.9 \\
300 & 3.3 & $Z$ & 2 & 2.2 & 1.1 \\
300 & 10 & $X$ & 1.8 & 2 & 2 \\
300 & 22 & $Y$ & 3 & 3.1 & 3.1 \\
\hline
\end{tabular}

$R_{\mathrm{S}}=0.00112 \cdot V+0.000138 \cdot V \cdot C$.

The results for the mean-kerf experiment are found in Table 4, and the ANOVA results are found in Table 5. For mean kerf, the voltage is the primary contributing factor (97.1\%, $p=0.0182)$, whereas both polar direction $(0.9 \%$, $p=0.6555)$ and capacitance $(0.2 \%, p=0.9112)$ play statistically insignificant roles, both of which are smaller than the residual noise in the data $(1.8 \%)$. We performed a regression analysis on mean kerf, based on the one input parameter that substantially influences mean kerf: voltage. The result, shown in Fig. 3, is an equation for mean kerf $K_{\mathrm{m}}\{\mu \mathrm{m}\}$ as a simple function of voltage $V$ volts that is a good fit in the region of the data collected:

$K_{\mathrm{m}}=0.203 \cdot V+104$.

The results for the standard-deviation-in-kerf experiment are found in Table 6, and the ANOVA results are found in Table 7. None of the input parameters play statistically significant roles 
Table 7 ANOVA for standard deviation in kerf

\begin{tabular}{lcccccc}
\hline Factor & df & Sum of squares & Mean square & $\boldsymbol{F}$ ratio & $\boldsymbol{p}$ value & Contribution (\%) \\
\hline Voltage & 2 & 0.1195 & 0.0598 & 0.5576 & 0.642 & 9 \\
Capacitance & 2 & 0.6862 & 0.3431 & 3.202 & 0.238 & 51.5 \\
Polar direction & 2 & 0.3114 & 0.1557 & 1.453 & 0.4077 & 23.4 \\
Residual & 2 & 0.2143 & 0.1072 & & & 16.1 \\
Total & 8 & 1.331 & & & \\
\hline
\end{tabular}

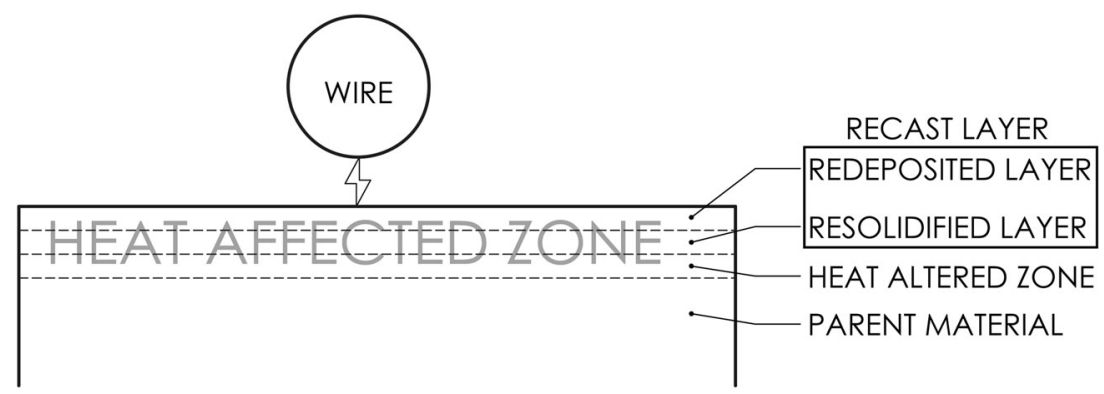

Fig. 4 Diagram of heat-affected zone (HAZ)

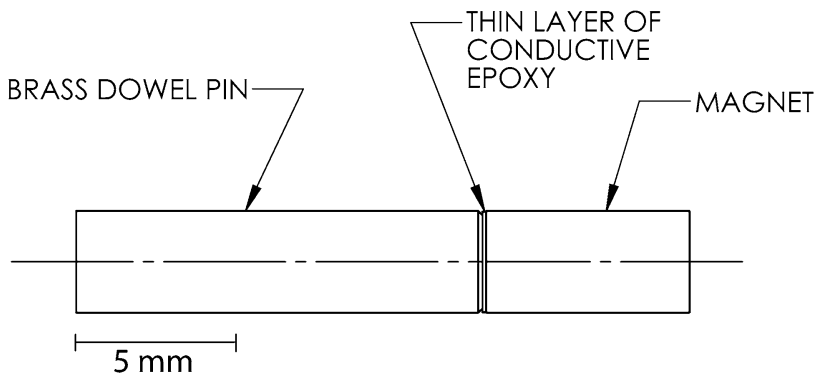

Fig. 5 Specimen for machining in rotary table

in the variation observed in kerf. For the entire set of 27 cuts made in this study, the standard deviation in kerf observed was always in the range of $1.1-3.1 \mu \mathrm{m}$, but with very little correlation with the input variables, so no regression model will be provided here.

\section{Loss of Magnetization due to WEDM Machining}

When a magnetic material is heated above its Curie temperature, it loses its magnetization. Since at least a portion of the WEDM NdFeB is heated above the Curie point out of necessity for material to be removed, there is a portion of the magnet that will become demagnetized. Figure 4 shows the various layers of the heat-affected zone (HAZ).

\subsection{Methods}

The following experiment was used to analyze the magnetic losses in the surface of the machined magnets. Five diametrically magnetized cylindrical magnets were machined using WEDM. The magnets had nominal dimensions of $3.175-\mathrm{mm}$ diameter and 6.350-mm length. The samples were fixtured for machining and testing by bonding them to the end of a brass dowel pin with electrically conductive adhesive as shown in Fig. 5. This fixturing allowed the wire to machine the entire

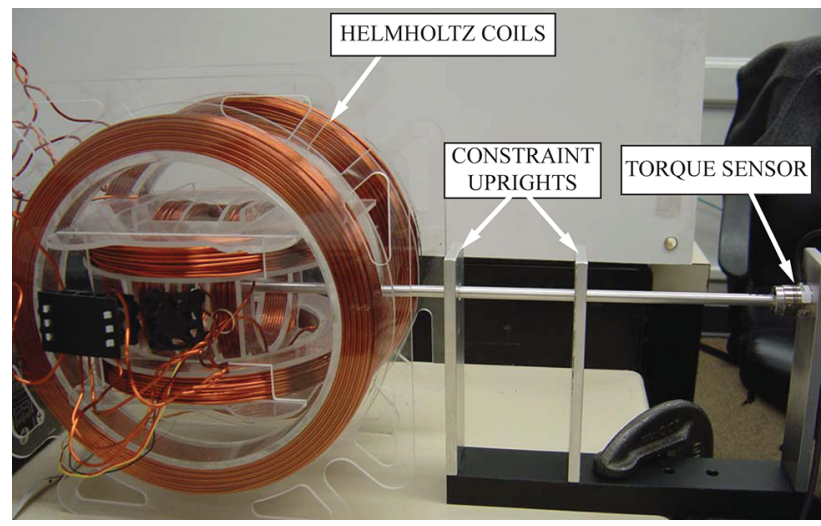

Fig. 6 Setup for torque measurements in Helmholtz coils. Constraint uprights are for overload protection, and do not contact shaft during experiments

length of the magnet, including part of the brass dowel. The adhesive used was Resinlab SEC1233 silver-filled epoxy with a volume resistivity of $0.003 \Omega \mathrm{mm}$. This added a negligible amount of resistance to the workpiece.

The samples were placed in a magnetic field generated by a set of orthogonal Helmoltz coils, shown in Fig. 6, and described in (Ref 20). The samples were fixed at the end of a long shaft attached to an ATI Nano 17 force-torque sensor (ATI Industrial Automation). The samples were placed in the common center of the coils, where the field is considered uniform. The coils created a magnetic field that rotated about the axis of the cylindrical magnet. A rotating field generates a sinusoidal torque on the magnet, as the measured torque \{newton-meter\} is the cross product of the magnet's total magnetic moment $\left\{\right.$ amp-meter $\left.{ }^{2}\right\}$ and the applied field $\{$ tesla\}. Five cylindrical magnets were measured with the force-torque sensor. The magnets were then machined to smaller diameters using WEDM, using a voltage of $300 \mathrm{~V}$ and a capacitance of $22 \mathrm{nF}$. The magnets were then re-measured in the coils with the force-torque sensor after machining. A field with magnitude 

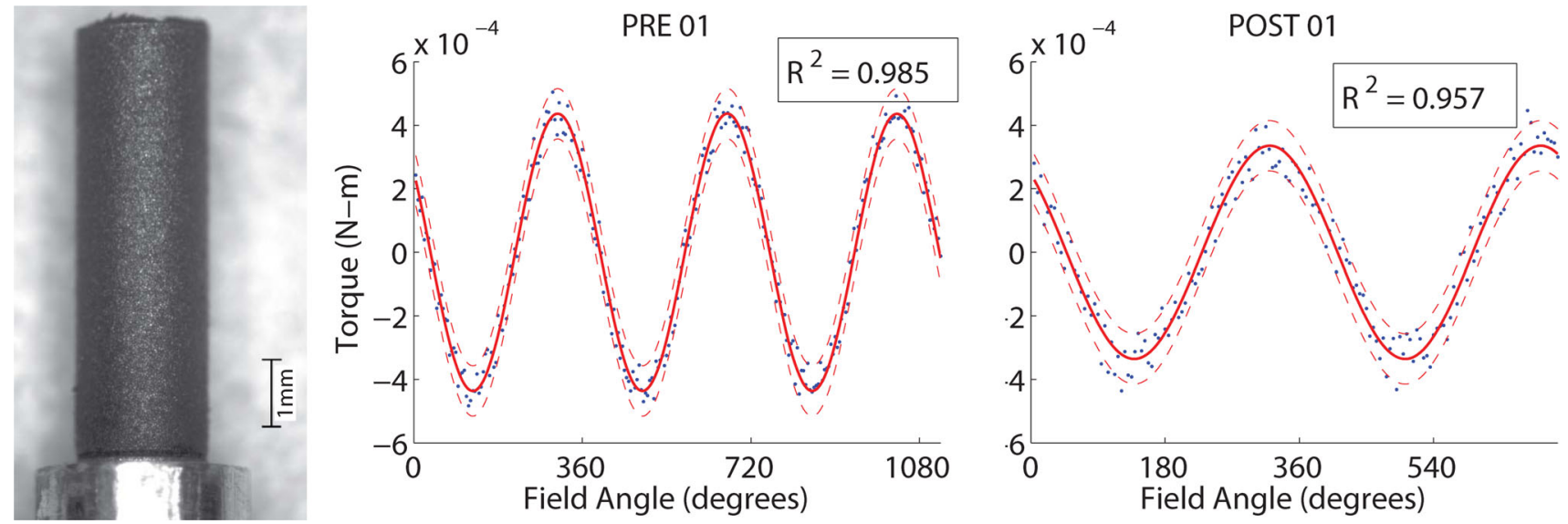

Fig. 7 Example WEDM sample for torque testing, along with its pre- and post-machined measured torques. Sinusoidal curve fits to the torque data are shown along with $R^{2}$ value and bands indicating two standard deviations

$10.37 \pm 0.05 \mathrm{mT}$ was applied and rotated at a speed of $0.025 \mathrm{~Hz}(40 \mathrm{~s} / \mathrm{rev})$ as torque data was collected. The measured torque curves were normalized and fit with a sine function using a least-squares fitting process. A characteristic magnet sample, after machining, is shown in Fig. 7, along with its preand post-machining measured magnetic torques.

The reduction in the magnitude of measured torque on the machined samples is due to the reduction in size from the bulk removal of magnetic material, the change in shape anisotropy (e.g., the reduction in diameter-to-length ratio, with a corresponding increase in the internal self-demagnetizing field), and the demagnetized surface layer due to the HAZ. The first two effects can be accounted for with known methods. We can combine the reduction in measured torque with FEA simulations to determine the depth of the demagnetized layer. Although a magnetic material may not have a homogeneous magnetic structure at microscopic scales, the material can be modeled as having an average magnetization across the volume. This average magnetization is a function of the shape of the material, magnetic history of the material, and the applied field; for hard-magnetic materials like $\mathrm{NdFeB}$ placed in a relatively weak magnetic field (i.e., where the applied field is much less than the coercive field required to demagnetize the permanent magnet: $\left.\left\|\vec{H}_{\text {int }}\right\|<<\left\|\vec{H}_{\mathrm{c}}\right\|\right)$, the average magnetization can be described by:

$\vec{M}_{\text {avg }}=\chi \vec{H}_{\text {int }}+\vec{M}_{\mathrm{r}}$

where $\chi$ is the susceptibility of the material, $\vec{H}_{\text {int }}$ is the internal field, and $\vec{M}_{\mathrm{r}}$ is the magnetic remanence, which is the shape-corrected magnetization remaining after magnet manufacturing and magnetization (Ref 21). The internal field is a function of the applied field and the demagnetizing field created by the magnet itself:

$\vec{H}_{\text {int }}=\vec{H}_{\text {app }}+\vec{H}_{\text {demag }}$.

The demagnetization field is a function of geometry and material magnetization and can be written as:

$\vec{H}_{\text {demag }}=-N \vec{M}_{\text {avg }}$

where $N$ is the demagnetization factor in the direction of magnetization, which is a function of geometry. Combining Eqs. (3-5), the average magnetization can be described as a function of applied field and remnant magnetization by:
$\vec{M}_{\mathrm{avg}}=\frac{1}{1+N \chi}\left(\chi \vec{H}_{\mathrm{app}}+\vec{M}_{\mathrm{r}}\right)$.

The torque experienced by the permanent magnetic material in an external field is then:

$\vec{\tau}=\mu_{0} V\left(\vec{M}_{\text {avg }} \times \vec{H}_{\text {app }}\right)$

where $V$ is the magnetized volume and $\mu_{0}=4 \pi \times 10^{-7} \mathrm{Tm} / \mathrm{A}$ is the permeability of free space. Because any vector crossed with itself is equal to zero, Eq. (7) reduces to:

$\vec{\tau}=\frac{\mu_{0} V}{1+N \chi}\left(\vec{M}_{r} \times \vec{H}_{\text {app }}\right)$.

An FEA model was created using Ansoft ${ }^{\circledR}$ Maxwell ${ }^{\circledR}$ release 14.0 software to simulate the geometry and solve Eq. (3) to determine the thickness of the post-machined demagnetized layer. The analysis assumes a quasistatic solution to Maxwell's electricity and magnetism equations, and a demagnetized layer of uniform thickness due to heating in the WEDM process. The geometry modeled, shown in Fig. 8, consists of a cylinder of $\mathrm{NdFeB}$ magnetized diametrically and placed in a uniform magnetic field that is orthogonal to the remanent magnetization. The free variables in this analysis are the demagnetized layer thickness and the remanent magnetization. The cylinder length, cylinder diameter, and applied field strength are determined by measurement. Calibration of the remanent magnetization for the analysis is performed by recognizing the linearity in Eq. (3) and multiplying the manufacturer-supplied remanent magnetization by the ratio of measured pre-machining torque to FEA-calculated torque for each sample. For these calculations the demagnetized layer thickness is taken to be zero and the overall diameter is reduced to account for the nominal plating thickness of $18 \mu \mathrm{m}$ on the exterior of the magnet.

The demagnetized layer thickness is determined by modeling the post-machining geometries as a cylinder of magnetized $\mathrm{NdFeB}$ with remanent magnetization surrounded by a shell of $\mathrm{NdFeB}$ with no remanent magnetization as shown in Fig. 8. The diameter of the magnetized $\mathrm{NdFeB}$ is the measured diameter of the sample less the demagnetized layer thickness. The torque is calculated for each sample using FEA simulations at five different demagnetized layer thicknesses. The measured torques are then subtracted from the FEA torques, giving a 
modeling torque error for each sample at each assumed demagnetization layer. The interpolated value of demagnetized layer thickness that results in no error between the modeled

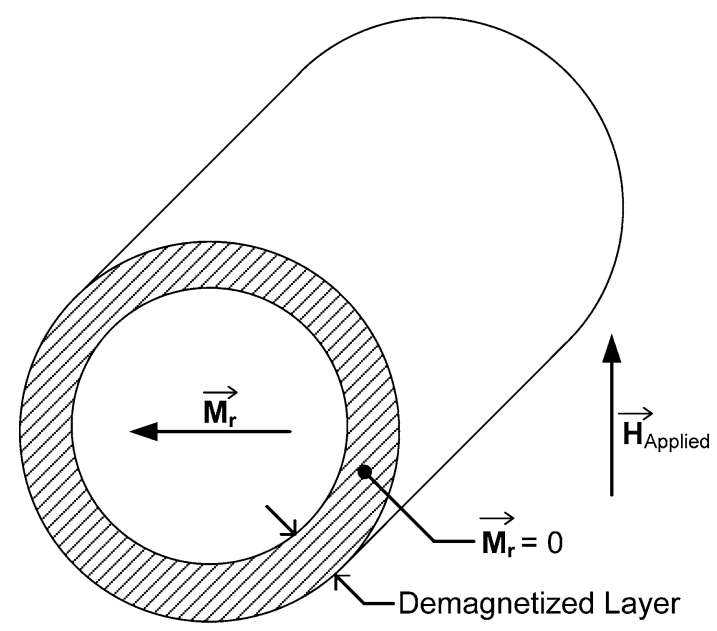

Fig. 8 FEA modeled geometry torque and the measured torque can be taken as the best estimate for the true thickness of the demagnetized layer.

\subsection{Results}

Table 8 provides, for each of the five samples: the measured length; the measured diameter, pre- and post-machining; the measure torque, pre- and post-machining, taken from the amplitude of the sinusoidal fits as in Fig. 7; and the calculated remanent magnetization for each sample, based on the premachining torque and geometry. The uncertainty reported includes the uncertainty in the measured torque, the applied field, and the thickness of the nickel-copper-nickel plating.

Figure 9 shows the difference between the measured torque and the FEA-modeled torque at five different modeled demagnetization-layer thicknesses, for each of the five samples, along with a least-squares fit line and tolerance bands. The tolerance bands are determined by combining the uncertainties in remanent magnetization, measured torque, and measured applied field. By analyzing the zero crossing of the least-squares fit line, as well as the tolerance bands, we determine the demagnetized layer thickness to be $35 \pm 15 \mu \mathrm{m}$.

Table 8 Measured diameter, length, and magnetic torque values with uncertainty, pre- and post-WEDM, with remanent magnetization estimated before machining

\begin{tabular}{|c|c|c|c|c|c|c|}
\hline $\begin{array}{l}\text { Sample } \\
\text { number }\end{array}$ & $\begin{array}{c}\text { Length } \\
(\mathrm{mm} \pm 1 \mu \mathrm{m})\end{array}$ & $\begin{array}{c}\text { Pre-Dia. } \\
(\mathbf{m m} \pm 1 \mu \mathrm{m})\end{array}$ & $\begin{array}{c}\text { Post-Dia. } \\
(\mathrm{mm} \pm 1 \mu \mathrm{m})\end{array}$ & $\begin{array}{l}\text { Pre-torque } \\
\left(10^{-3} \mathrm{Nm}\right)\end{array}$ & $\begin{array}{l}\text { Post-torque } \\
\left(10^{-3} \mathrm{Nm}\right)\end{array}$ & $\begin{array}{c}\text { Remanent } \\
\text { magnetization } \\
\left(1^{3} \mathbf{A} / \mathbf{m}\right)\end{array}$ \\
\hline 1 & 6.351 & 3.15 & 2.805 & $0.4364 \pm 0.0103$ & $0.3354 \pm 0.0108$ & $859.5 \pm 22.7$ \\
\hline 2 & 6.351 & 3.157 & 2.532 & $0.4410 \pm 0.0102$ & $0.2772 \pm 0.0107$ & $864.3 \pm 22.9$ \\
\hline 3 & 6.327 & 3.134 & 2.3 & $0.4291 \pm 0.0106$ & $0.2237 \pm 0.0104$ & $855.8 \pm 24.3$ \\
\hline 4 & 6.344 & 3.141 & 1.979 & $0.4123 \pm 0.0103$ & $0.1537 \pm 0.0103$ & $817.8 \pm 21.0$ \\
\hline 5 & 6.344 & 3.15 & 1.631 & $0.4400 \pm 0.0103$ & $0.1119 \pm 0.0104$ & $866.9 \pm 22.9$ \\
\hline
\end{tabular}

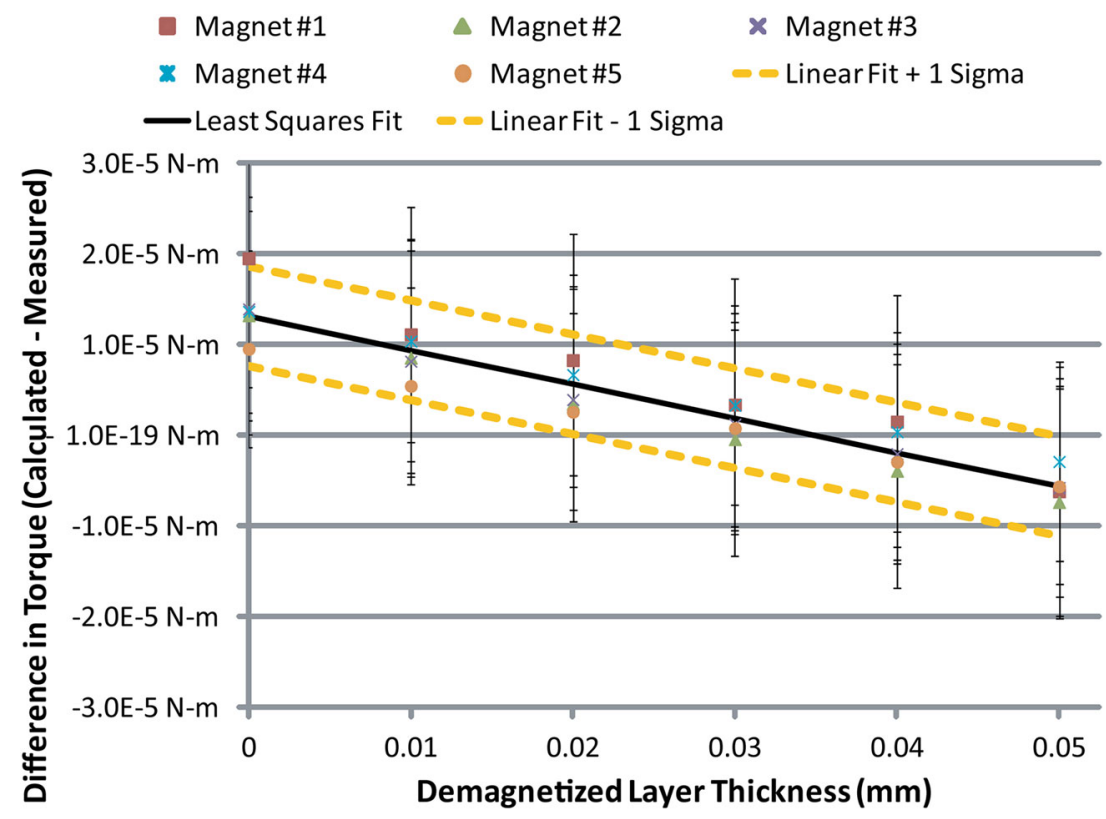

Fig. 9 FEA results for torque as a function of the thickness of the demagnetized layer 

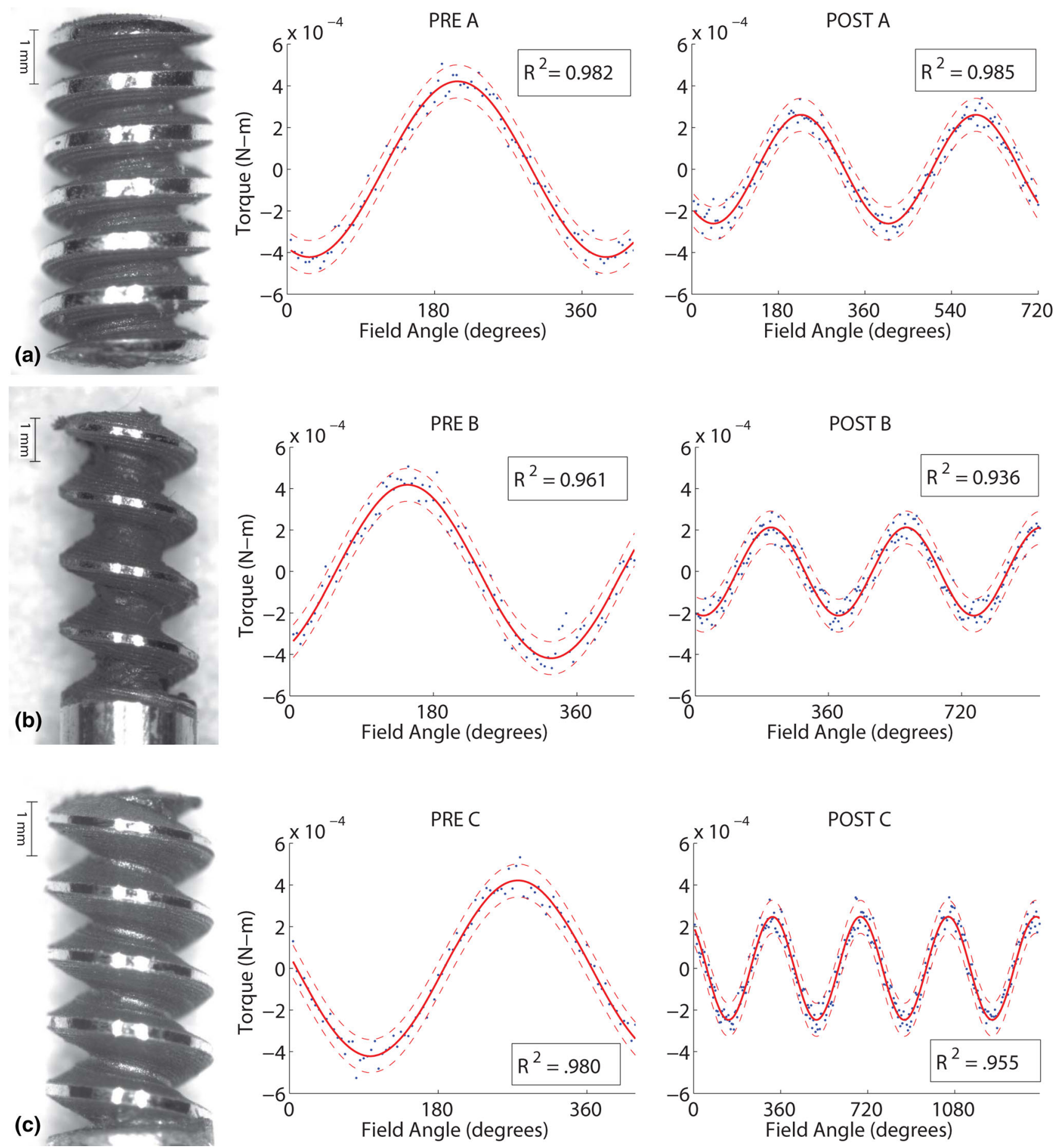

Fig. 10 WEDM screw prototypes, along with pre- and post-machined measured torques. Sinusoidal curve fits to the torque data are shown along with $R^{2}$ value and bands indicating two standard deviations

\section{Discussion}

The models for slicing rate and mean kerf presented in Sect. 2.2 are only explicitly valid for WEDM voltages in the range of 150-300 V, and for WEDM capacitances in the range of 3.3$22 \mathrm{nF}$. However, because simple models were proposed (i.e., the data was not overfit), it is reasonable to assume that the models could be extrapolated somewhat for values outside of these ranges. The results for slicing rate agree with published research (Ref 22) in the basic sense that increased spark energy density, or discharge energy (via increased capacitance and voltage) increases material removal rate. A limiting factor for WEDM at high energy discharge settings is high wire breakage rates. Higher discharge energies also cause higher surface roughness (Ref 23) and may cause higher loss of magnetic flux density (Ref 13).

The demagnetization results of Sect. 3.2 can only be strictly assumed to apply for the voltage $(300 \mathrm{~V})$ and capacitance 
(22 nF) used. Since it is reasonable to assume that the demagnetized-layer thickness would only tend to decrease with a decrease in voltage or capacitance, assuming a demagnetized-layer thickness of $35 \pm 15 \mu \mathrm{m}$ is likely a conservative estimate of magnetization loss if lower values of voltage or capacitance are used. It may be possible to regain the magnetization in the outer demagnetized layer by remagnetizing the sample after machining is complete, but that is beyond the scope of this work. Reductions in EDM NdFeB magnetic flux have reported as much as $3 \mathrm{~mm}$ below the surface at high discharge energies (Ref 13); however, because the machine used was of the MOSFET-type, and because of the way that the magnetization data was collected and interpreted, it is difficult to make a direct comparison of our result to this significantly larger reported value. There is evidence that control of the internal temperature of the $\mathrm{NdFeB}$ workpiece (e.g., through dielectric fluid flushing) can be used to help keep magnetization intact (Ref 14).

Several different screw prototype designs have been machined with WEDM cutting parameters of $300 \mathrm{~V}$ and $22 \mathrm{nF}$, three of which are shown, along with their pre- and post-machining measured torques, in Fig. 10. The torque data show that for screws machined from $\mathrm{NdFeB}$ permanent magnets, significant torque can still be generated relative to the original torque in the magnet, to propel and guide the screws. Note that a reduction in torque is expected, even in the absence of any demagnetization in the HAZ, simply due to large amount of material removal. Additional details on the fabrication of the screws can be found in Greer (Ref 19).

One aspect that complicated machining of the magnet workpieces (both the magnetic screws and the samples in the original experiment) was that the machined particles adhered to the part. The micro-EDM jet flushing installed on the machine enhanced the removal of these particles, but did not solve the problem completely. The adhered particles contributed to an increased number of shorts detected by the machine, which in turn led to an increase in machining times. The machining times reported in this paper include these effects. Furthermore, when attempting to remove the wire from deep machined slots, the wire would wedge in the particles and often break. It was obvious that the relatively gentle flushing action that is typically used for micro-EDM provided insufficient flushing velocity to remove debris before it could reattach itself to the workpiece. Future work will include a coaxial flushing system that operates at higher pressure and velocity. The coaxial jet, in contrast to the side flushing of the original micro-EDM system, should immediately carry the debris out of the cutting zone, thereby preventing the cutting problems and wire breakages that were observed originally. It is likely that machining times of $\mathrm{NdFeB}$ would decrease if such changes to the flushing system were implemented.

\section{Conclusions}

This paper considered wire electrical discharge machining (WEDM) of magnetized sintered NdFeB permanent magnets, using an RC-type machine. Using a Taguchi design of experiments and subsequent analysis of variance, it was found that (1) WEDM voltage and capacitance affect slicing rate, (2) WEDM voltage affects mean kerf, (3) no parameters tested effect the variation in kerf with any statistical significance, and
(4) the polarity direction of the magnet being machined has no effect on the machining process with any statistical significance. Regression analysis was used to provide simple equations that describe slicing rate and mean kerf as a function of voltage and capacitance; the results apply explicitly for voltages in the range of $150-300 \mathrm{~V}$ and capacitances in the range of 3.3-22 $\mathrm{nF}$, but the simple models likely extrapolate somewhat beyond these explicit ranges.

A finite element analysis was performed, in combination with experiments, to determine the depth of the demagnetized layer from heating effects from the WEDM. This analysis found the depth of the layer that lost its magnetization was $35 \pm 15 \mu \mathrm{m}$. This result applies for a WEDM voltage of $300 \mathrm{~V}$ and capacitance of $22 \mathrm{nF}$, and will be a conservative estimate for lower voltages and capacitances, based on previously published trends.

Three screw prototypes of varying geometry were manufactured using WEDM parameters of $300 \mathrm{~V}$ and $22 \mathrm{nF}$. Torque measurements taken before and after machining reveal that a significant portion of the magnetic torque remains after the machining process. This provides evidence that permanentmagnet devices such as magnetic screws can be successfully manufactured from commercially available $\mathrm{NdFeB}$ magnets.

\section{Acknowledgment}

This material is based upon work supported by the National Science Foundation under Grant Nos. 0952718 and 0654414.

\section{References}

1. B.J. Nelson, I.K. Kaliakatsos, and J.J. Abbott, Microrobots for Minimally Invasive Medicine, Ann. Rev. Biomed. Eng., 2010, 12(1), p 55-85

2. N. Luo, N. Zhang, and C.D. Graham, Variation of Hardness with Temperature in Sintered NdFeB Magnets, J. Appl. Phys., 1987, 61(8), p 3442-3444

3. A. Kruusing, S. Leppävuori, A. Uusimäki, B. Petrêtis, and O. Makarova, Micromachining of Magnetic Materials, Sens. Actuators $A, 1999$, 74(1-3), p 45-51

4. L. Li, D. Wang, Z.W. Niu, and J.H. Zhang, Investigation of Material Removal Mechanism in EDM of Sintered NdFeB Permanent Magnet, Key Eng. Mater., 2007, 334-335, p 937-940

5. L. Li, R. Gou, and G. Yuan, A New Erosion Protection Machining Method for Sintered NdFeB Permanent Magnet, J. Aust. Ceram. Soc., 2008, 44(1), p 31-33

6. S.F. Miller, A.J. Shih, and J. Qu, Investigation of the Spark Cycle on Material Removal Rate in Wire Electrical Discharge Machining of Advanced Materials, Int. J. Mach. Tools Manuf., 2004, 44, p 391-400

7. L. Li, D. Wang, Z.W. Niu, and Z.Y. Li, EDM with USM Combination Process of Sintered NdFeB Permanent Magnet, Adv. Mater. Res., 2007, 24-25, p 139-143

8. L. Li, Z.W. Niu, and G. Yuan, Effect of Ultrasonic Vibration of Tool on Electrical Discharge Machining of Sintered NdFeB Magnet, Prog. Mach. Technol., 2009, 407-408, p 628-631

9. P.J. Ross, Taguchi Techniques for Quality Engineering: Loss Function, Orthogonal Experiments, Parameter and Tolerance Design, McGrawHill, New York, 1988

10. P. Campbell, Permanent Magnet Materials and their Application, Cambridge University Press, Cambridge, 1994

11. J.M.D. Coey, Rare-Earth Iron Permanent Magnets, Oxford University Press, Oxford, 1996

12. S.F. Miller, C.-C. Kao, A.J. Shih, and J. Qu, Investigation of Wire Electrical Discharge Machining of Thin Cross-Sections and Compliant Mechanisms, Int. J. Mach. Tools Manuf., 2005, 45, p 1717-1725 
13. H. Takezawa, Y. Ichimura, T. Suzuki, T. Muramatsu, and N. Mohri, Relationship Between Thermal Influence and Magnetic Characteristics in Electrical Discharge Machining of Magnetic Materials, Key Eng. Mater, 2012, 516, p 575-579

14. Y. Ichimura, H. Takezawa, and N. Mohri, Relationship Between Magnetic Flux Density and Temperature Distributions of Permanent Magnets by EDM, Key Eng. Mater., 2012, 523-524, p 322-327

15. C. Orino, D. Rakwal, J.D. Jorgensen, and E. Bamberg, Development of a precision, low-cost, small footprint wire electron discharge machine (WEDM), Proc. ASPE Conf., 2006, p 239-242

16. D. Rakwal and E. Bamberg, Slicing, Cleaning and Kerf Analysis of Germanium Wafers Machined by Wire Electrical Discharge Machining, J. Mater. Process. Technol., 2009, 209(8), p 3740-3751

17. D. Rakwal, S. Heamawatanachai, P. Tathireddy, F. Solzbacher, and E. Bamberg, Fabrication of Compliant High Aspect Ratio Silicon Microelectrode Arrays Using Micro-wire Electrical Discharge Machining, Microsyst. Technol., 2009, 15(5), p 789-797
18. D. Rakwal, and E. Bamberg, Using Taguchi Methods to Optimize the Slicing Rate and Surface Roughness for Wire Electrical Discharge Machining of p-type Germanium, Proc. 15th Int Symp Electromachining (ISEM XV), Pittsburg, PA, 2007, p 127-132

19. J. Greer, Wire Electrical Discharge Machining of Helical Devices from Permanent Magnets. Master's Thesis, Department of Mechanical Engineering, University of Utah, Salt Lake City, Utah, 2011

20. A.W. Mahoney, J.C. Sarrazin, E. Bamberg, and J.J. Abbott, Velocity Control with Gravity Compensation for Magnetic Helical Microswimmers, Adv. Robotics, 2011, 25(8), p 1007-1028

21. R.C. O'Handley, Modern Magnetic Materials: Principles and Applications, Wiley, New York, 2000

22. L. Li, G. Yuan, Z. Niu, and R. Hou, Effects of Electrical Discharge Energy on Machining Performance of Sintered NdFeB Magnet, Mater. Sci. Forum, 2009, 620-622, p 711-714

23. L. Li, Z. Niu, F. Yin, and Y. Liu, Surface Integrity of Sintered NdFeB Permanent Magnet After EDM, Adv. Mater. Res., 2012, 503-504, p 27-30 